\title{
1 The Effects of Ocean Surface Waves on Global Forecast 2 in CFS Modeling System v2.0
}

3 Ruizi Shi ${ }^{1}$, Fanghua $\mathrm{Xu}^{1}$, Li Liu ${ }^{1}$, Zheng $\mathrm{Fan}^{1}$, Hao $\mathrm{Yu}^{1}$, Xiang $\mathrm{Li}^{2}$ and Yunfei Zhang ${ }^{2}$

$4 \quad{ }^{1}$ Ministry of Education Key Laboratory for Earth System Modeling, and Department of Earth System

5 Science, Tsinghua University, Beijing 100084, China

$6{ }^{2}$ Key Laboratory of Marine Hazards Forecasting, National Marine Environmental Forecasting Center,

7 Ministry of Natural Resources, Beijing, 100081, China

8 Correspondence to: Fanghua Xu (xu@mail.tsinghua.edu.cn)

9 Abstract. It has been well known that ocean surface gravity waves have enormous effects on physical

10 processes at the atmosphere-ocean interface. However, the effects of surface waves on global forecast

11 in several days are less studied. To investigate this, we incorporated the WAVEWATCH III model into the Climate Forecast System Model version 2.0 (CFS2.0), with the Chinese Community Coupler version 2.0 (C-Coupler2). Two major wave-related processes, the Langmuir mixing and the sea surface momentum roughness, were considered. Extensive comparisons were performed against in-situ buoys, satellite measurements and reanalysis data, to evaluate the influence of the two processes on the forecast of sea surface temperature, mixed layer depth, significant wave height, and 10-m wind speed. A series of 7-day simulations demonstrate that the newly developed atmosphere-ocean-wave coupling system could improve the CFS global forecast. The Langmuir mixing parameterization could increase the vertical movement of water and effectively reduce the warm bias of sea surface temperature and shallow bias of mixed layer depth in the Antarctic circumpolar current in austral summer, whereas the significant wave height and $10-\mathrm{m}$ wind speed are insensitive to it. On the other hand, the modified momentum roughness length could significantly reduce the overestimated $10-\mathrm{m}$ wind speed and significant wave height in mid-high latitudes. This is because the enhanced frictional dissipation at high wind speed could 
reduce $10-\mathrm{m}$ wind speed and consequently decrease the significant wave height. But its effect on the temperature structure in upper ocean is less obvious.

\section{Introduction}

Ocean surface gravity waves play an important role in modifying physical processes at the atmosphereocean interface, which can influence momentum, heat, and moisture fluxes across the air-sea interface (Li and Garrett 1997; Taylor and Yelland, 2001; Moon et al., 2004; Belcher et al., 2012; Moum and Smyth, 2019). For instance, ocean surface waves can modify the ocean surface roughness to influence the marine atmospheric boundary layer and thus change the momentum, latent heat, and sensible heat transfer (Taylor and Yelland, 2001; Moon et al., 2004). The breaking waves inject turbulent kinetic energy in the upper ocean, which can enhance the mixing process (Terray et al. 1996). Nonbreaking surface waves can also affect mixing in the upper ocean by adding a wave-related Reynolds stress (Qiao et al., 2004). The wave-related Stokes drift interacts with the Coriolis force and produces the CoriolisStokes force (Hasselmann 1970). The shear of Stokes drift is a critical reason for the generation of Langmuir circulation, which could significantly deepen the mixed layer by strong vertical mixing process both at climate scale (Li and Garrett 1997; Belcher et al., 2012) and at weather scale (Kukulka et al., 2009). If sea ice is present, the interaction of wave, ocean and atmosphere is further complicated (Kohout and Meylan, 2008; Squire et al., 2009).

As Fox-Kemper et al. (2019) expected, the improvement to atmosphere-ocean coupling with a better presentation of the effects of surface gravity waves, is one of the challenges and focuses in ocean modeling for the next decade. Regional coupled models were developed to study tropical cyclones, storm surge and other coastal processes at small or medium scales (e.g. Prakash et al., 2018; Ricchi et al., 2017; 
Pianezze et al., 2018; Wu et al., 2019). The Coupled Ocean-Atmosphere-Wave-Sediment Transport Modeling System (COAWST) developed by Warner et al. (2010) is one of well-known fully-coupled models, which includes effects of wave-state-dependent ocean surface roughness, radiation stress, bottom stress and Stokes drift-related processes. The COAWST has been well applied in various locations such as the South China Sea (Sun et al., 2019; Wu et al., 2019), Bay of Bengal (Prakash et al., 2018) and Mediterranean (Ricchi et al., 2017). On the other hand, the coupled models with a wave component at global scale were primarily developed for long-term climate research (e.g. Qiao et al. 2010; Breivik et al. 2015; Chune, et al. 2018; Fan et al., 2012; Fan and Griffies, 2014; Li et al. 2016, 2017). The effects of waves on short term forecast at global scale have been considered negligible for long time. Since the impact of wave-related processes is important not only for the synoptic processes but also for the frequent interactions at multiple spatial scales as aforementioned, it is of great interest to investigate the effects of surface ocean waves on short-term forecast in a global atmosphere-ocean-wave system with suitable presentations of wave-related processes.

To realize a fully-coupled modeling system, establishing suitable connections between the wave component and the atmosphere/ocean component are crucial. In coupled systems, commonly the atmosphere and ocean components provide 10-m winds and surface currents, sometimes with other variables such as sea surface temperature and water depth, to the wave model as forcing fields (Chen et al. 2007; Warner et al. 2010; Breivik et al. 2015; Li et al. 2016; Pianezze et al., 2018). Compared to a single wave model, in which the inputted reanalysis datasets usually have an interval more than 3 hours, the forcing fields in the wave component have a finer time interval (Fan et al., 2012). Meanwhile, the wave component sends wave parameters, such as wave length, period and significant wave height, to the atmosphere and ocean components. These wave parameters could be used in various wave-related 
parameterizations. In this study, we coupled the WAVEWATCH III to the Climate Forecast System Model (CFS) using the Chinese Community Coupler version 2.0 (C-Coupler2). We mainly considered two effects induced by waves at the ocean-atmosphere interface, surface roughness and Langmuir cells induced mixing. This is because both processes have strong influences on momentum and energy fluxes across the air-sea interface and could effectively improve the simulation results (e.g. Fan et al., 2012; Fan and Griffies, 2014; Li et al. 2016, 2017). Four series of 7-day forecasts were produced with this system. The performance of the system was then compared with observations and reanalysis data. Sensitivity experiments with various wave parameterizations were carried out to evaluate the contributions of surface roughness and Langmuir mixing to the changes of atmosphere and ocean. In addition, the performance of various wave parameterizations was evaluated as well. The analysis is structured as follows: methods and a set of experiments with various parameterizations are described in Section 2; the observation and reanalysis data are introduced in Section 3, and the results of experiments are evaluated against these available data in Section 4; a summary and discussion follow in Section 5.

\section{Methods and Experiments}

\subsection{Coupling WAVEWATCH III with CFS2.0}

The version 5.16 of WAVEWATCH III (WW3; WAVEWATCH III Development Group, 2016) developed by the National Oceanic and Atmospheric Administration/National Centers for Environmental Prediction (NOAA/NCEP) has been incorporated into the Climate Forecast System Model, version 2.0 (CFS2.0; Saha et al., 2014) as a new model component. The latitude range of WW3 is $78^{\circ} \mathrm{S}-78^{\circ} \mathrm{N}$ with a spatial resolution of $1 / 3^{\circ}$; the frequency range is $0.04118-0.4056 \mathrm{~Hz}$ and the total number of frequencies is 25 ; the number of wave directions is 24 with a resolution of $15^{\circ}$; the maximum global time step is 450 
$\mathrm{s}$ and the minimum source term time step is $300 \mathrm{~s}$. The CFS contains two components, the global forecasting system (GFS; details about the GFS are available at http://www.emc.ncep.noaa.gov/GFS/doc.php) as the atmosphere component and the modular ocean model version 4 (MOM4; Griffies et al., 2004) as the ocean component. The MOM4 is integrated on a nominal $0.5^{\circ}$ horizontal grid with enhanced horizontal resolution in the tropics, and has 40 vertical levels; the vertical spacing is $10 \mathrm{~m}$ in the upper $225 \mathrm{~m}$, and then increases in unequal intervals to the bottom at $4478.5 \mathrm{~m}$. The GFS uses a spectral triangular truncation of 126 waves (T126) in the horizontal, which is equivalent to a grid resolution of nearly $100 \mathrm{~km}$, and 64 sigma-pressure hybrid layers in the vertical. The time steps of both MOM4 and GFS are $180 \mathrm{~s}$.

This coupled system uses the Chinese Community Coupler version 2.0 (C-Coupler2; Liu et al., 2018) for interpolating and passing variables between its atmosphere, ocean, and wave components, to guarantee each component receives inputs and supplies outputs on its own grid. The C-Coupler2 is a common, flexible and user-friendly coupler, which contains dynamic 3-D coupling system and enables variables to remain conserved after interpolation. The variables are exchanged every other time step, which in atmosphere and ocean components is $180 \mathrm{~s}$, and in wave component is $450 \mathrm{~s}$.

A schematic diagram of the coupled atmosphere-ocean-wave system is shown in Fig. 1. As illustrated, WW3 is two-way coupled with MOM4 and GFS, through the C-Coupler2. WW3 is forced by 10-m wind from GFS and sea surface current from MOM4, and then generates and evolves the wave action density spectrum. Meanwhile, the momentum roughness length is passed to GFS from WW3 (see section 2.3), and the surface Stokes drift velocity is passed to MOM4 (see section 2.2). In this study, both the CFS and WW3 use warm boots; the daily initial fields at 00:00 for CFS are generated by the real time operational Climate Data Assimilation System (CDAS; Kalnay et al., 1996), downloaded from the CFS 
111 WW3, a single WW3 model is set up synchronously (see section 2.4).

112

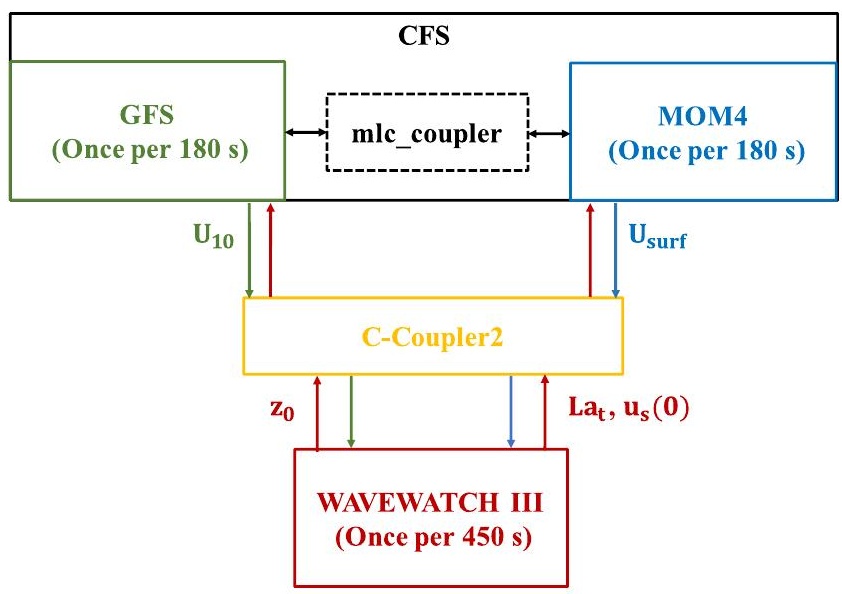

Figure 1. A schematic diagram of the atmosphere-ocean-wave coupled modeling system. The arrows indicate the coupled variables that are passed between the model components. In the diagram, $\mathrm{z}_{0}, \mathrm{La}_{\mathrm{t}}, \mathrm{u}_{\mathrm{s}}(0), \mathrm{U}_{10}$, and $\mathrm{U}_{\text {surf }}$ are momentum roughness length, turbulent Langmuir number, surface Stokes drift velocity, 10-m wind and surface current, respectively.

\subsection{Parameterizations of Langmuir Mixing}

\subsubsection{McWilliams and Sullivan (2000) Parameterization}

McWilliams and Sullivan (2000) improved the turbulent velocity scale W in KPP by introducing an enhancement factor $\varepsilon$, to account for both boundary layer depth changes and nonlocal mixing by Langmuir turbulence. In their work, they indicated $\mathrm{W}\left(\mathrm{W}=k u_{*} / \phi\right.$, where $u_{*}$ is the surface friction velocity, $\phi$ is the dimensionless flux profile, and $k=0.4$ is the von Kármán constant) varies in proportion to the turbulent Langmuir number, that is,

$$
\begin{gathered}
\mathrm{W}=\frac{k u_{*}}{\phi} \varepsilon, \\
\varepsilon=\sqrt{1+0.08 L{a_{\mathrm{t}}^{-4}}^{-4}}
\end{gathered}
$$




$$
L a_{\mathrm{t}}=\sqrt{\frac{u_{*}}{\left|u_{s}(0)\right|}}
$$
and waves. They suggested a projected Langmuir number,

$$
\begin{gathered}
L a_{\text {proj }}=\sqrt{\frac{u_{*} \cos (\alpha)}{\left|u_{S}(0)\right| \cos \left(\theta_{w w}-\alpha\right)}}, \\
\alpha \approx \tan ^{-1}\left[\frac{\sin \theta_{w w}}{\frac{u_{*}}{u_{S}(0) k} \ln \left(\left|\frac{h}{z_{1}}\right|\right)+\cos \theta_{w w}}\right] .
\end{gathered}
$$
Here $\alpha$ is the angle between wind and Langmuir cell, $\theta_{w w}$ is the angle between Stokes drift and wind, and $Z_{1}$ is the four times of the significant wave height. In this case, Van et al. (2012) suggested the form of $\varepsilon$ should be 


$$
\varepsilon=|\cos \alpha| \sqrt{1+\left(3.1 L a_{\mathrm{proj}}\right)^{-2}+\left(5.4 L a_{\mathrm{proj}}\right)^{-4}} .
$$

139

140

141 length as

$$
z_{c h}=\frac{z_{0} g}{u_{*}^{2}}
$$
Here $z_{0}$ is the roughness length, and $z_{c h}=0.014$ is the constant Charnock number. The corresponding scatterplot of $z_{0}$ in GFS versus 10 -m wind speed is shown in Fig.2 (black dots). The $z_{0}$ in GFS increases relatively slowly with increasing wind speed, especially at high winds. 


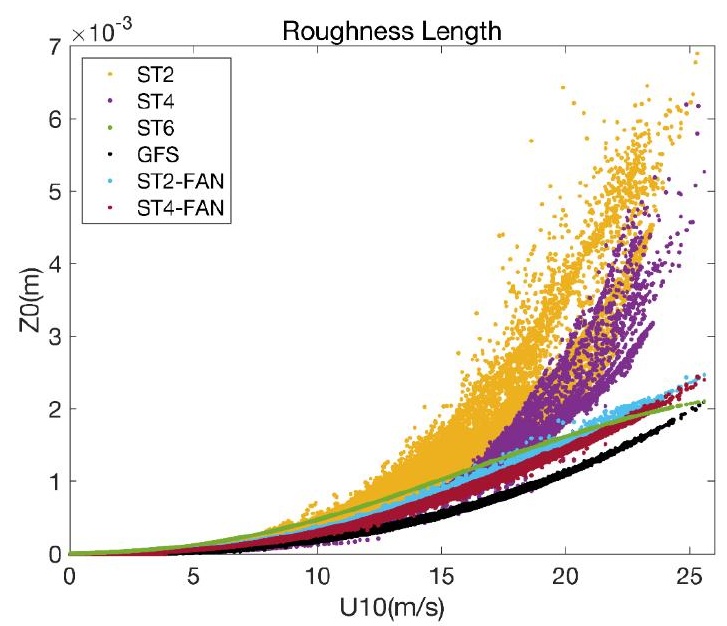

Figure 2. Scatterplot of momentum roughness length $z_{0}(m)$ in various source term packages versus $10-m$ wind speed $(\mathrm{m} / \mathrm{s})$.

In WW3, input of momentum and energy by wind, and dissipation for wave-ocean interaction are two important terms (combined as input-dissipation source term) in the energy balance equation (WAVEWATCH III Development Group, 2016). Several different packages to calculate the inputdissipation source term (ST) are offered in the WW3 version 5.16, and the most commonly used ones are ST2 (Tolman and Chalikov, 1996), ST3 (Janssen, 2004; Bidlot, 2012), ST4 (Ardhuin et al., 2010), and ST6 (Zieger et al., 2015). In ST4 package, wind speed is assumed to satisfy the traditional logarithmic profile in the neutral boundary layer

$$
U_{10}=\frac{u_{*}}{k} \log \left(\frac{z_{10}}{z_{0}}\right)
$$
where $U_{10}$ is $10-\mathrm{m}$ wind speed, and $z_{10}$ is the corresponding height $(10 \mathrm{~m})$. The same relationship is also used in ST2 package (Tolman and Chalikov, 1996). And the roughness length $z_{0}$ is obtained from this relationship in ST4 and ST2 (purple and yellow dots in Fig.2). Fan et al., (2012) indicated that the $z_{0}$ in ST2 increases rapidly with wind speed at high winds and results in the fast-increasing drag coefficient, which is inconsistent with the drag coefficient leveling off for extremely high wind speed in 


$$
\begin{gathered}
\frac{z_{0} g}{u_{*}^{2}}=a\left(\frac{c_{p i}}{u_{*}}\right)^{b}, \\
a=\frac{0.023}{1.0568^{U_{10}}}, b=0.012 U_{10},
\end{gathered}
$$

where $c_{p i}$ is the phase speed of dominant wind-forced waves.

In this study, we chose the ST4 package to calculate the input and dissipation term, since ST4 has shown the best performance in the simulation of significant wave height (SWH) at global scale (compared in section 2.4), which is consistent with the study of Stopa et al (2016). Fan et al. (2012)'s parameterization was then applied in ST4 (referred to as ST4-FAN) to obtain new $z_{0}$. The estimates of $z_{0}$ from ST2FAN and ST4-FAN are shown in blue and dark red dots of Fig.2, respectively. The fast-rising trend of $z_{0}$ at high wind speed is obviously restrained. And $z_{0}$ from ST4-FAN is generally smaller than that from ST2-FAN.

\subsection{Initialization of WAVEWATCH III}

The initial wave fields were generated from the 10-day simulations starting from rest in a single WW3 model. To minimize the biases of the initial wave fields, we ran simulations with ST2, ST3, ST4, and 

wind forcing, which generated the minimum SWH bias (Table S1 in the supplementary), was applied to generate initial wave fields for all experiments listed in Table 1.

Table 1. List of Numerical Experiments

\begin{tabular}{ccc}
\hline Experiments & WW3 to MOM4 & WW3 to GFS \\
\hline CTRL & None & None \\
VR12-AL-ONLY & VR12-AL parameterization & None \\
Z0-ONLY & None & $z_{0}$ from ST4 \\
VR12-AL-Z0 & VR12-AL parameterization & $z_{0}$ from ST4 \\
MS2K-Z0 & MS2K parameterization & $z_{0}$ from ST4 \\
VR12-AL-Z0-FAN & VR12-AL parameterization & $z_{0}$ from ST4-FAN \\
\hline
\end{tabular}
parameterizations and momentum roughness lengths on the ocean and atmosphere in four 7-day periods, January 3 to 10, 2017, July 1 to 8, 2018, August 3 to 10, 2018, and January 1 to 8, 2019. results of CFS in CTRL are consistent with the corresponding CFS Reanalysis data (Saha et al., 2010). 


\section{Data}

Sea surface temperature (SST), profiling temperature and salinity, 10-m wind speed (WSP10), and significant wave height $(\mathrm{SWH})$ from observations and reanalysis datasets are used to evaluate the simulation results.

The daily average satellite Optimum Interpolation SST (OISST) data is obtained from the National Oceanic and Atmospheric Administration (NOAA), with $0.25^{\circ} \times 0.25^{\circ}$ resolution (Reynolds et al., 2007; https://www.ncdc.noaa.gov/oisst). The global Argo observational profiles of temperature and salinity (Li et al., 2019) is from China Argo Real-time Data Center (www.argo.org.cn). The fifth generation European Centre for Medium-Range Weather Forecasts (ECMWF) Reanalysis (ERA5) datasets of WSP10 and SWH with a spatial resolution of $0.5^{\circ}$ and a temporal resolution of 1 hour are also used (Hersbach and Dee, 2016; https://cds.climate.copernicus.eu/cdsapp\#!/dataset/ reanalysis-era5-single$\underline{\text { levels}) . ~ A d d i t i o n a l l y, ~ t h e ~ W S P 10 ~ a n d ~ S W H ~ o b s e r v a t i o n s ~ f r o m ~ t h e ~ a v a i l a b l e ~ N a t i o n a l ~ D a t a ~ B u o y ~ C e n t e r ~}$ (NDBC) buoy data (https://www.ndbc.noaa.gov), and the along-track SWH from Jason-3 satellite measurements (https://aviso-data-center.cnes.fr) Geophysical Data Record (GDR) with precise orbit and an orbital velocity of $7.2 \mathrm{~km} / \mathrm{s}$ are applied for comparison purposes. 

loss of generality, we compared the distributions of SST on the $4^{\text {th }}$ day as the intermediate state, and the similar distributions on the last day are also shown in Figs. S1\&S2 of the supplementary. Figure 3 and and percentage absolute difference of the bias from experiments versus the CTRL (Fig. 3c-g and Fig. 4cas SST in CTRL minus OISST. And to highlight the differences of other experiments versus the CTRL,

238 the percentage absolute differences (PAD) of the bias are computed as PAD $=\frac{\left|\widehat{y_{S}}-y\right|-\left|\widehat{y_{C}}-y\right|}{|y|} \times 100 \%$, where $y$ is OISST, $\widehat{y_{c}}$ is simulated SST in CTRL and $\widehat{y_{s}}$ is simulated SST in other experiments (Fig. vice versa. 

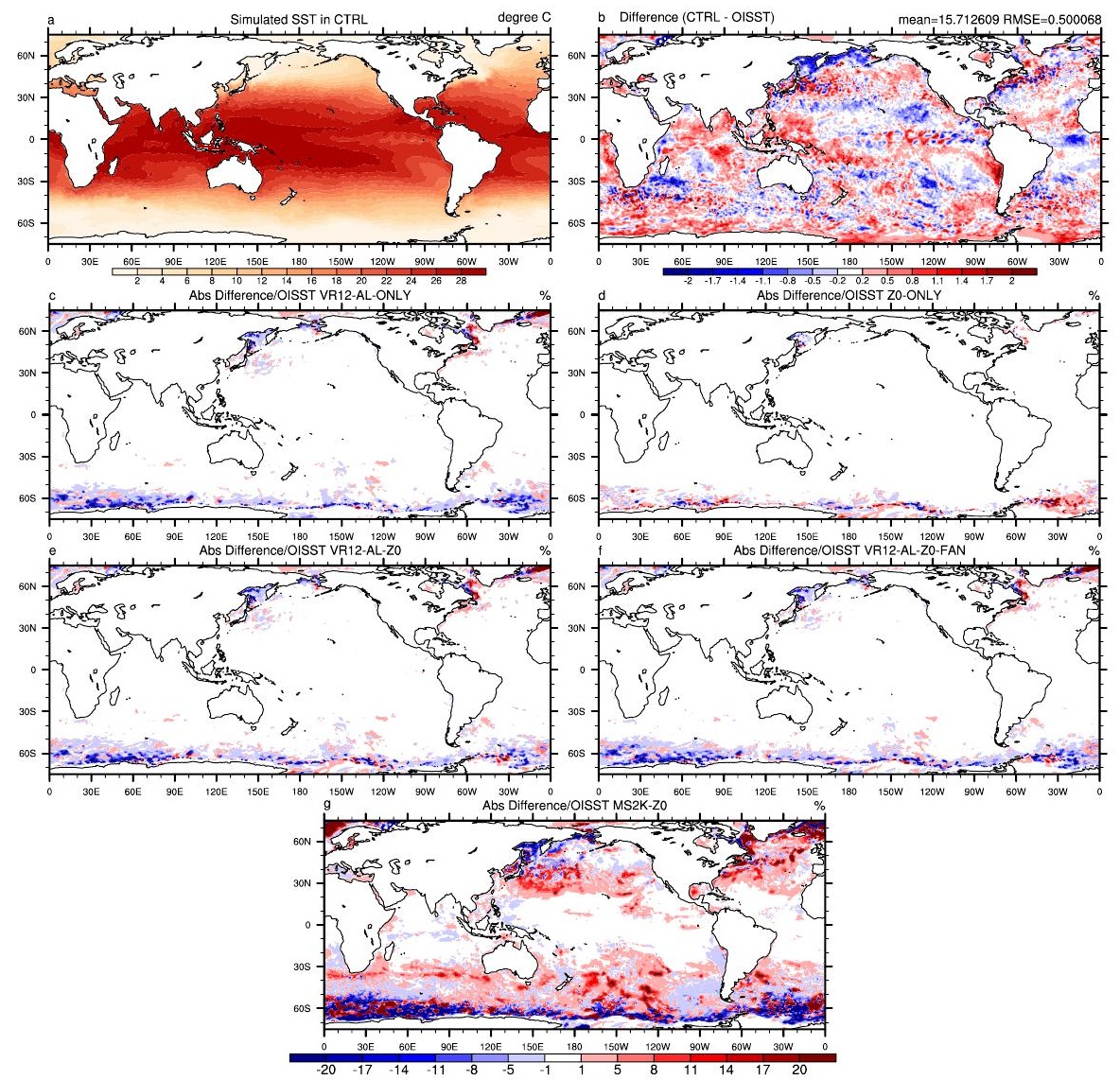

Figure 3. The daily average SST $\left({ }^{\circ} \mathrm{C}\right)$ in $\mathrm{CTRL}$, its bias in CTRL and percentage absolute difference of bias on

January 7, 2017: a the SST in CTRL, $\mathbf{b}$ the SST bias between CTRL and OISST (CTRL minus OISST), $\mathbf{c} / \mathbf{d} / \mathbf{e} / \mathbf{f} / \mathbf{g}$ the percentage absolute difference between VR12-AL-ONLY/Z0-ONLY/VR12-AL-Z0/VR12-AL-Z0-FAN/MS2K-Z0 and CTRL. The absolute difference is a percentage computed as PAD $=\frac{\left|\widehat{y_{s}}-y\right|-\left|\widehat{y_{c}}-y\right|}{|y|} \times 100 \%$, where $y$ is OISST, $\widehat{y_{c}}$ is simulated SST in CTRL and $\widehat{y_{s}}$ is simulated SST in other experiments, so a negative value means that the error is smaller than that of CTRL, and vice versa. 

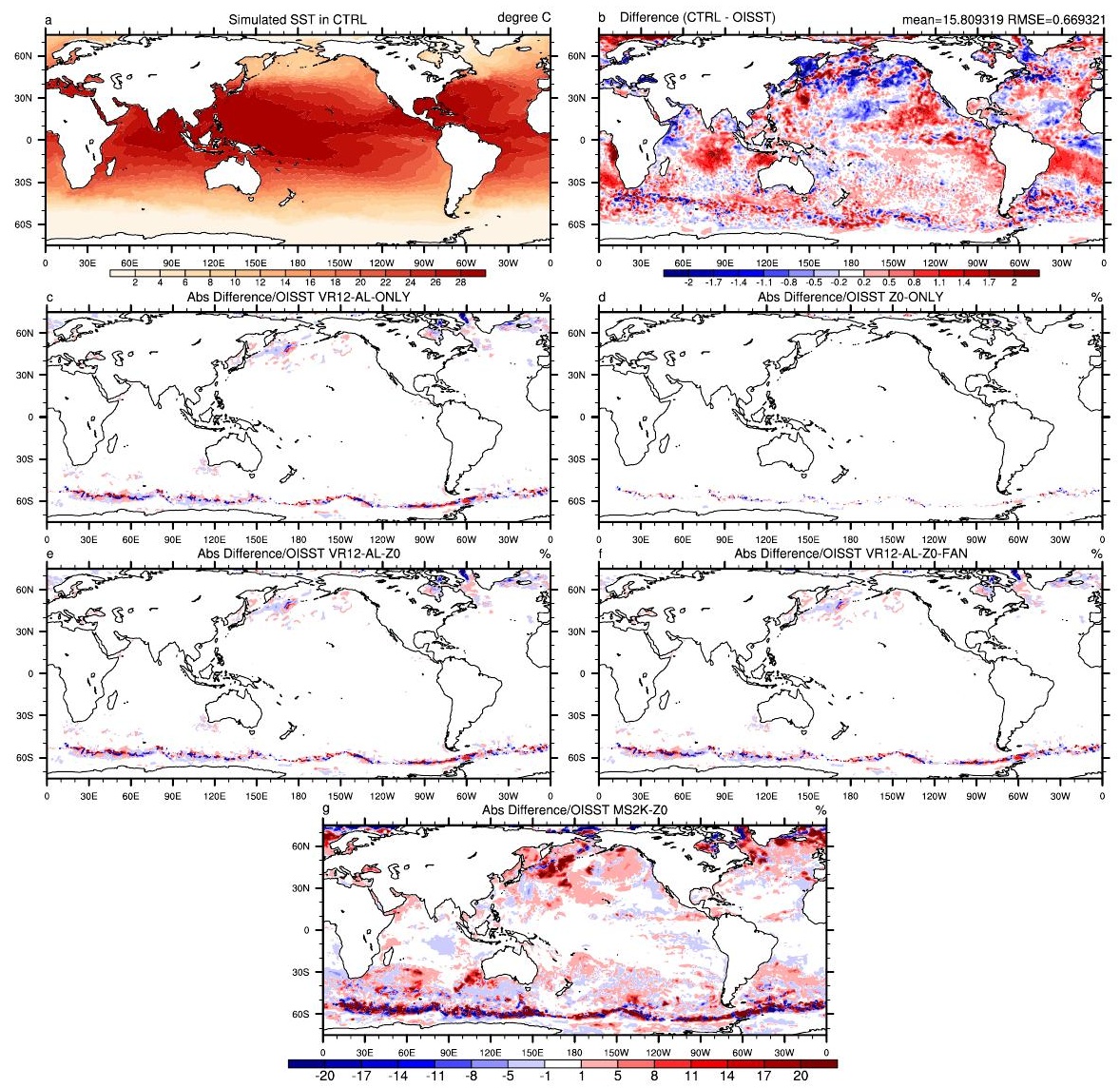

Figure 4. As Fig. 3, but for the daily average SST $\left({ }^{\circ} \mathrm{C}\right)$ in CTRL, its bias in CTRL and absolute difference of bias on August 7, 2018 

are almost identical to Fig. 3c, indicating that the SST is insensitive to the change of surface roughness induced by MS2K parameterization, which has cooled down the surface ocean greatly. As a result, although the warm bias in ACC is greatly reduced, the cold bias is enhanced in mid latitudes (Fig. 3g). Compared to the simulations in January, the simulations with VR12-AL parameterization in August show of the reduced warm bias during austral winter in the south of $50^{\circ} \mathrm{S}$ (Fig. 4b). These results are consistent with the studies of Belcher et al. (2012) and Li et al. (2016), which indicated that the improvements of simulation by Langmuir mixing parameterizations in Southern Ocean are obvious mainly in austral summer but not winter. To examine the robustness of these variations, two tests from January 1 to 8 , 2019 and from July 1 to 8, 2018 were conducted. The results are in good agreement with the previous simulations (Figs. S7\&S8 in the supplementary). Similarly, the SST in August also becomes too cold in MS2K-Z0 (Fig. 4g), especially in the mid latitude regions of the Northern Hemisphere, where the SST is already underestimated in CTRL (Fig. 4b). 

$\left(0-360^{\circ} \mathrm{E}, 45-78^{\circ} \mathrm{S}\right)$ are shown in Fig. 5. Both the MS2K (dark red) and VR12-AL (yellow, blue and 

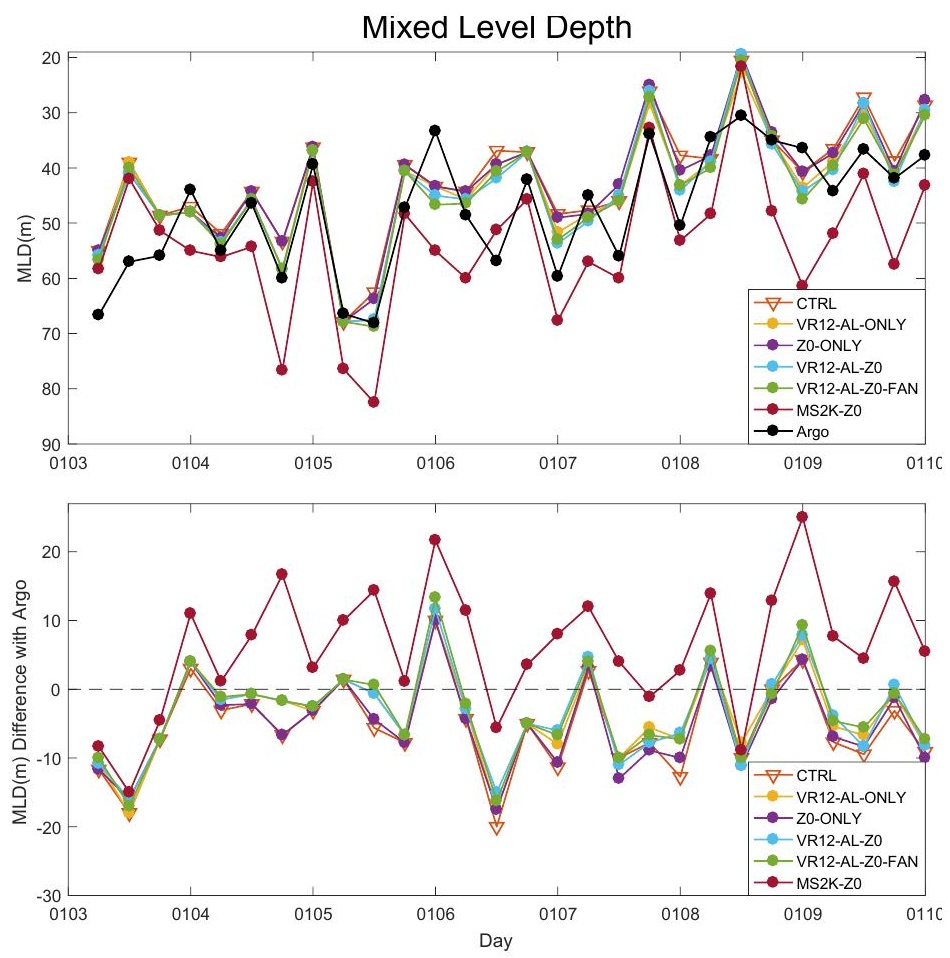

Figure 5. The time series of domain-averaged $\left(0-360^{\circ} \mathrm{E}, 45-78^{\circ} \mathrm{S}\right)$ mixed layer depth (MLD; m; upper panel) and MLD bias versus Argo profile data (simulations minus Argo; lower panel). The time intervals are 6 hours.

\subsection{Significant Wave Height (SWH) and Wind Speed at $10 \mathrm{~m}$ (WSP10)}

To evaluate the performance of the wave simulation, the simulated SWHs were compared with Jason-3 GDR along-track quality-checked altimeter measurements and the ERA5 reanalysis data. Here we compared the Jason-3 data and simulations at 00:00 (the Jason-3 data within 20 min from 00:00 were applied), and interpolated the simulated SWHs at 00:00 onto the satellite orbit. The 7-day averaged SWH correlation coefficients, skill scores and RMSEs from 00:00 on Jan 3, 2017 to 00:00 on Jan 10, 2017 are documented in Table 2. Compared with CTRL, experiments with coupled $z_{0}$ show improvements to the SWH simulation, especially the VR12-AL-Z0-FAN. Although the improvements are not quite large, because those are the global average results, whereas the significant improvements mainly distribute in 

2018, the correlation coefficient, skill score and RMSE of CTRL are $0.79,0.86$ and 0.68 , and those of of which the RMSE is 0.61 and reduces $10.0 \%$.

Table 2. 7-day Averaged Correlation Coefficient, RMSE and Skill Score of SWH in Simulations and ERA5 versus the Jason-3 Observation from 00:00 on Jan 3, 2017 to 00:00 on Jan 10, 2017. Bold marks represent the highest correlation coefficient, the lowest RMSE and the highest skill score (except ERA5); the RMSE and skill score (SS) are calculated as RMSE $=\sqrt{\sum_{i=1}^{n}\left(\widehat{y_{l}}-y_{i}\right)^{2} / n}$ and SS $=1-\frac{\sum_{i=1}^{n}\left(\widehat{y}_{l}-y_{i}\right)^{2}}{\sum_{i=1}^{n}\left(\hat{y}_{l}-\bar{y}_{l}|+| y_{i}-\bar{y}_{l}\right)^{2}}$, respectively, where $\widehat{y_{l}}$ is simulated value or ERA5 data, $y_{i}$ is Jason-3 observation and $\bar{y}_{l}$ is the average, $\mathrm{i}=1, \mathrm{n}$ and $\mathrm{n}$ is the total number of

\begin{tabular}{cccc}
\hline & $\begin{array}{c}\text { Correlation Coefficient } \\
(\mathrm{P}<0.01)\end{array}$ & RMSE & Skill Score \\
\hline CTRL & 0.85 & 0.60 & 0.91 \\
VR12-AL-ONLY & 0.85 & 0.61 & 0.90 \\
Z0-ONLY & 0.85 & 0.58 & 0.91 \\
VR12-AL-Z0 & 0.85 & 0.58 & 0.91 \\
MS2K-Z0 & 0.82 & 0.60 & 0.89 \\
VR12-AL-Z0-FAN & $\mathbf{0 . 8 6}$ & $\mathbf{0 . 5 7}$ & $\mathbf{0 . 9 2}$ \\
ERA5 & 0.87 & 0.51 & 0.92 \\
\hline
\end{tabular}



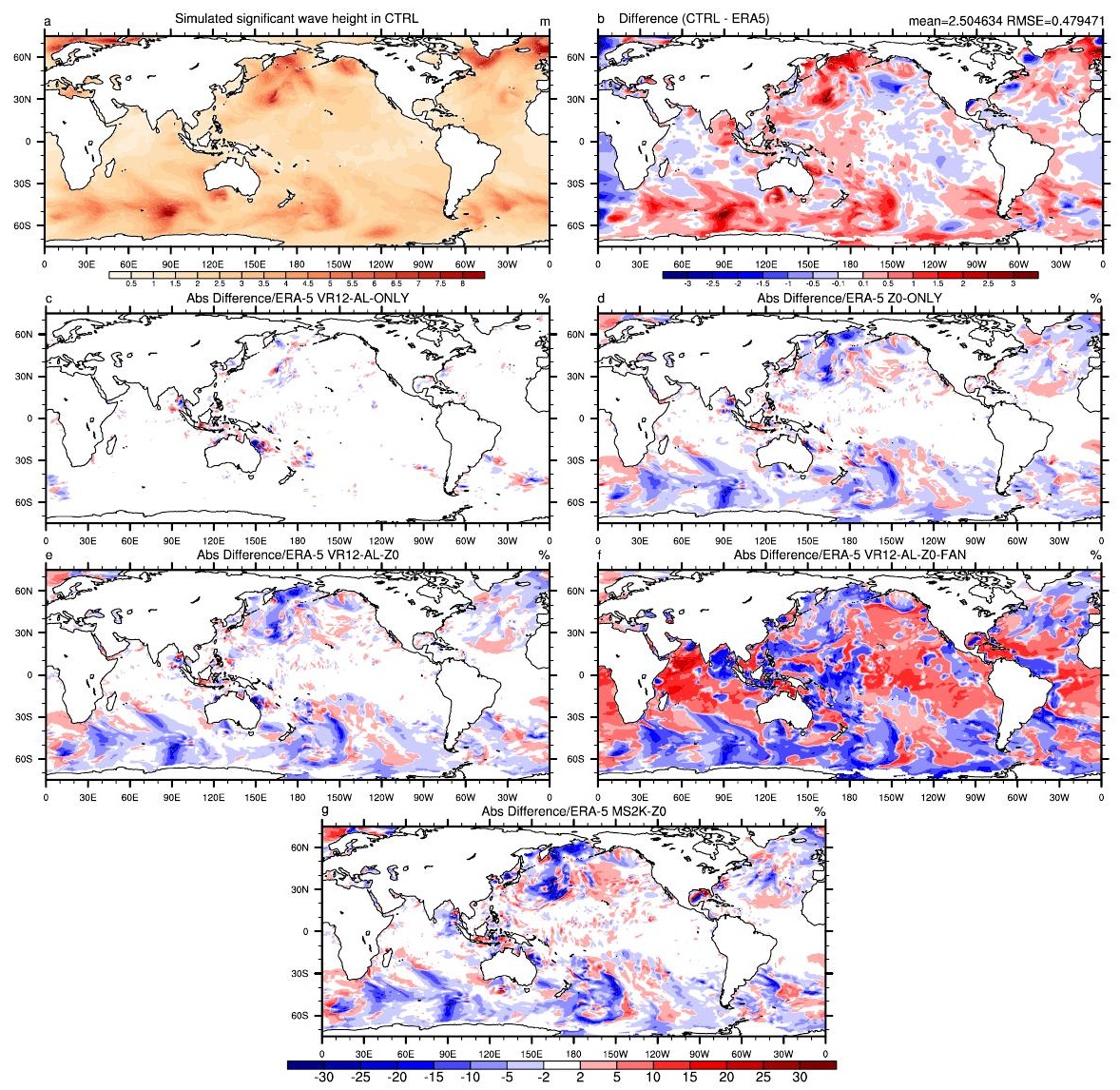

Figure 6. The SWH (m) in CTRL, its bias in CTRL and percentage absolute difference of bias on January 7, 2017:

a the SWH in CTRL, $\mathbf{b}$ the SWH bias between CTRL and ERA5 (CTRL minus ERA5), $\mathbf{c} / \mathbf{d} / \mathbf{e} / \mathbf{f} / \mathbf{g}$ the percentage absolute difference between VR12-AL-ONLY/Z0-ONLY/VR12-AL-Z0/VR12-AL-Z0-FAN/MS2K-Z0 and CTRL. The absolute difference is a percentage computed as $\mathrm{PAD}=\frac{\left|\widehat{y_{s}}-y\right|-\left|\widehat{y_{c}}-y\right|}{|y|} \times 100 \%$, where $y$ is the SWH from ERA5, $\widehat{y_{c}}$ is simulated SWH in CTRL and $\widehat{y_{s}}$ is simulated SWH in other experiments, so a negative value means that the error is smaller than that of CTRL, and vice versa. 

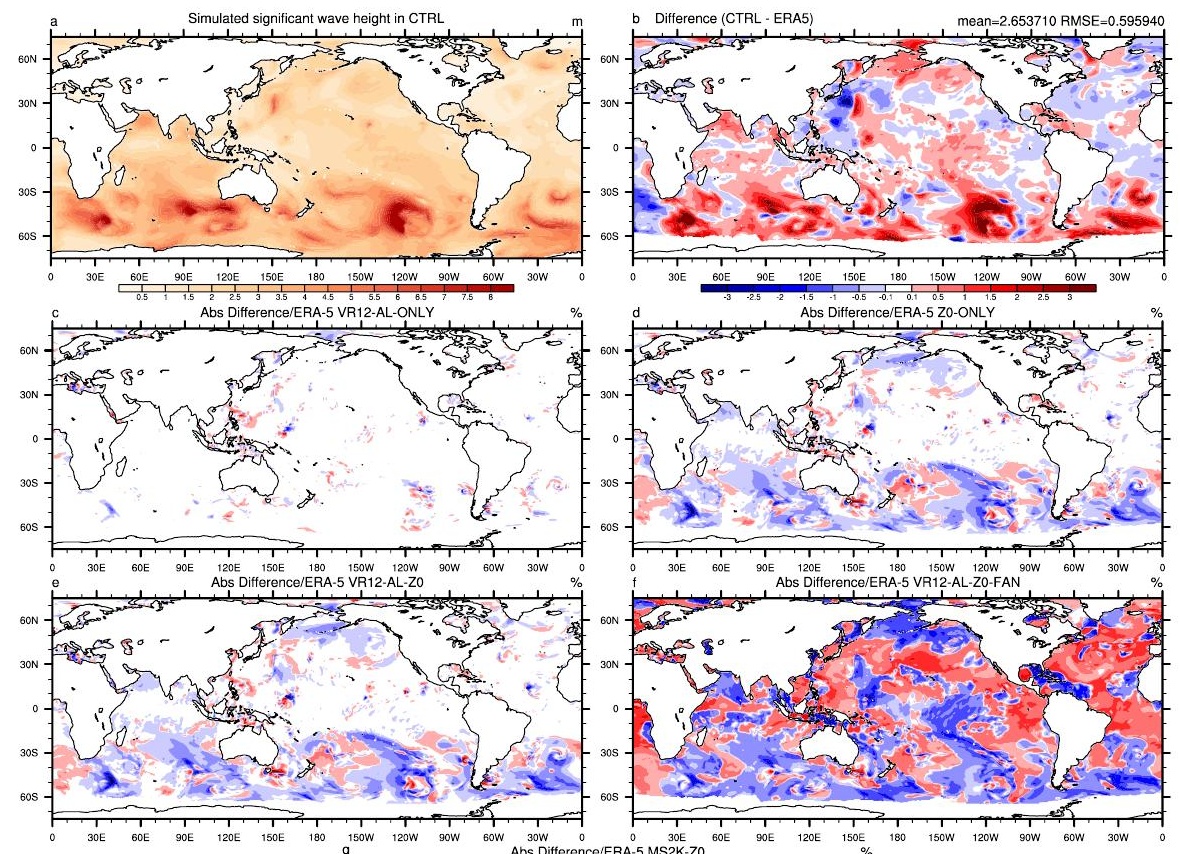

Figure 7. As Fig. 6, but for the SWH (m) in CTRL, its bias in CTRL and absolute difference of bias on August 7, 2018.

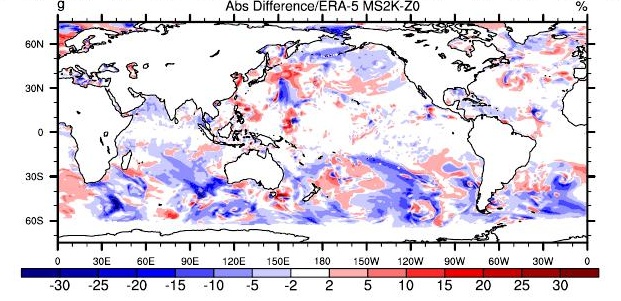

The waves in WW3 are mainly generated by the 10-m winds, since the effect of sea surface current is

much weaker. The comparisons of the WSP10 from numerical experiments with the ERA5 wind data

(Fig.8\&9) indicate that the overestimated WSP10 (red shaded areas in Figs.8b\&9b) could lead to the overestimated SWH (red shaded areas in Fig.6b\&7b). In Z0-ONLY and VR12-AL-Z0 (Fig.8d\&e; Fig.9d\&e), after introduced the $z_{0}$ from ST4 in GFS, the biases of overestimated WSP10 are reduced and so are the biases of the SWH. In VR12-AL-Z0-FAN, the decrease of WSP10 is slightly weaker than those in VR12-AL-Z0 (Figs. 8f\&9f), due to the $z_{0}$ from ST4-FAN which is lower than that from ST4 



Figure 8. The WSP10 (m/s) in CTRL, its bias in CTRL and percentage absolute difference of bias on January 7 , 2017: a the 10-m wind in CTRL, $\mathbf{b}$ the 10-m wind bias between CTRL and ERA5 (CTRL minus ERA5), $\mathbf{c} / \mathbf{d} / \mathbf{e} / \mathbf{f} / \mathbf{g}$ the percentage absolute difference between VR12-AL-ONLY/Z0-ONLY/VR12-AL-Z0/VR12-AL-Z0-FAN/MS2K$\mathrm{Z} 0$ and CTRL. The absolute difference is a percentage computed as $\operatorname{PAD}=\frac{\left|\widehat{y_{s}}-y\right|-\left|\widehat{y_{c}}-y\right|}{|y|} \times 100 \%$, where $y$ is 

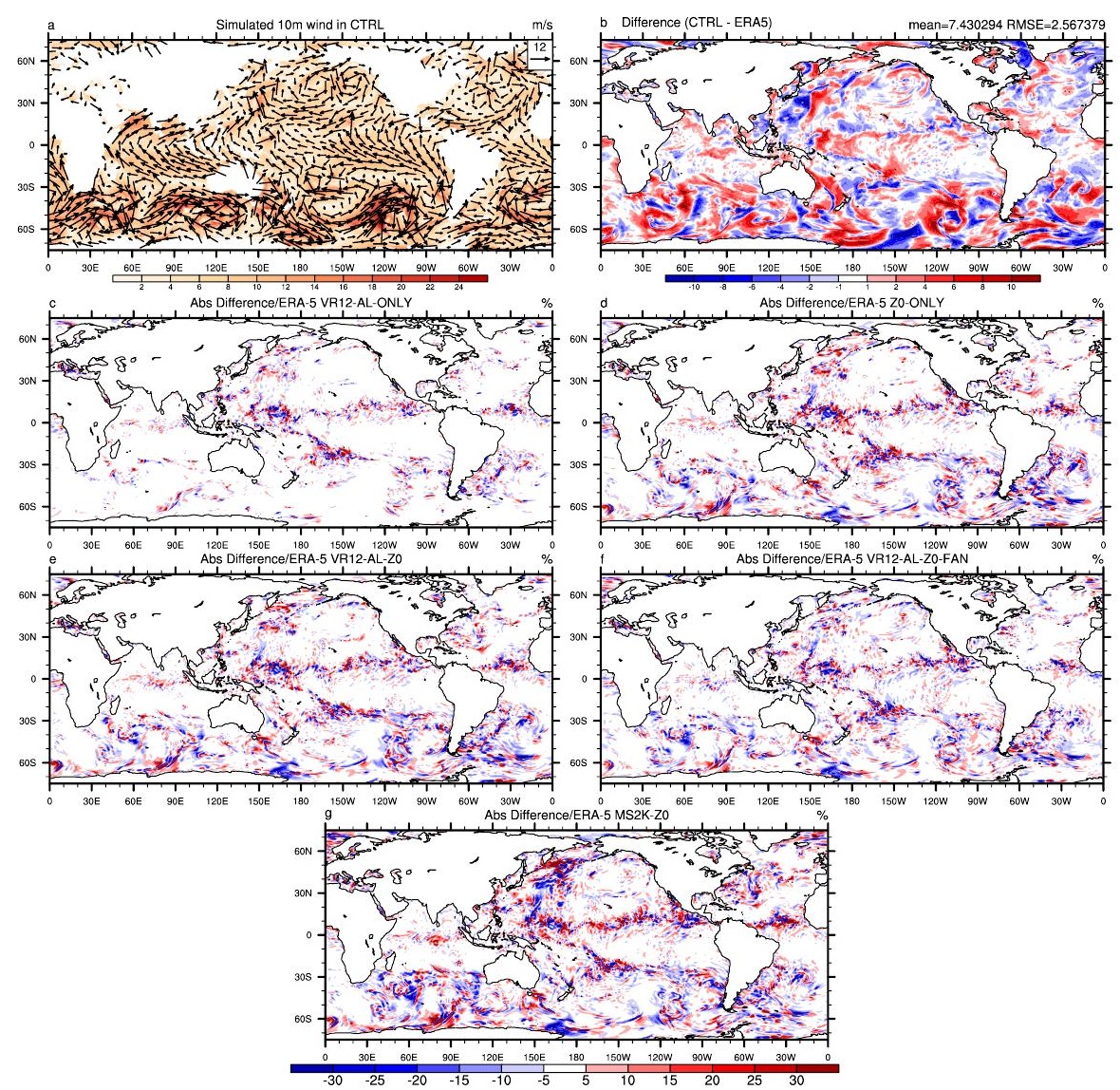

Figure 9. As Fig. 8, but for the WSP10 (m/s) in CTRL, its bias in CTRL and absolute difference of bias on August 7, 2018.

To demonstrate the relationship of SWH and WSP10 more clearly, we calculated the 7-day mean absolute percentage error (MAPE) for SWH and WSP10 between simulation results and NDBC buoy data (locations shown in Fig. 10). In general, the difference of SWH corresponds well to the difference of WSP between CTRL and ERA5 (shaded areas in Fig. 10). The lower the MAPE, the better the performance of the simulation. The corresponding MAPE differences compared with CTRL for the other 

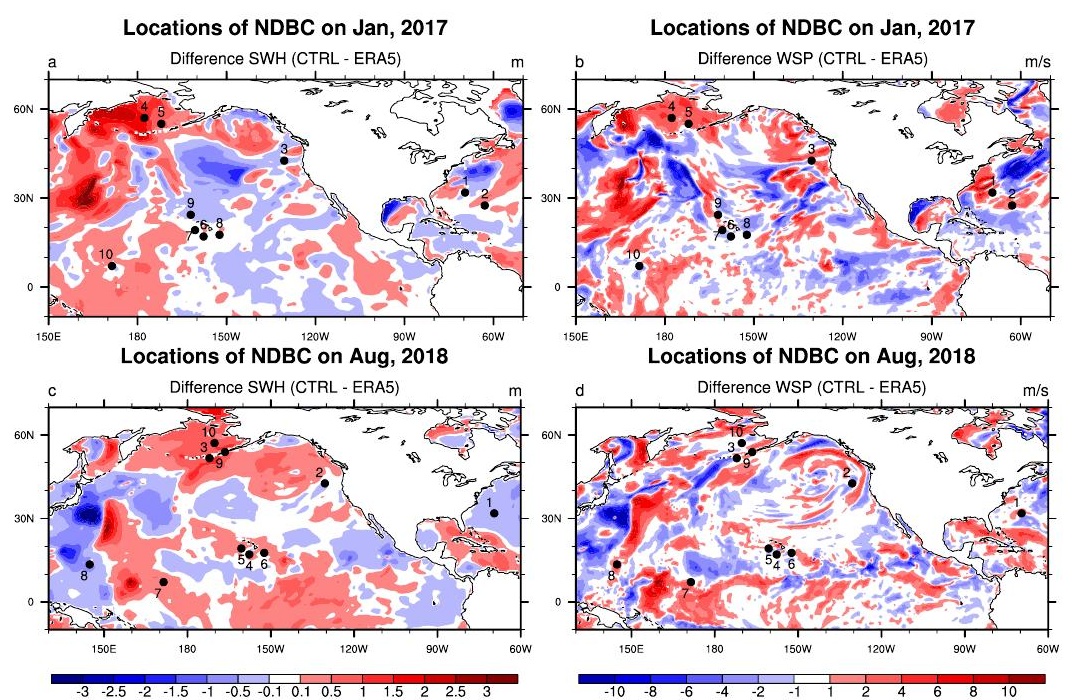

Figure 10. The locations of NDBC buoy data on Jan, 2017 (a, b) and Aug, 2018 (c, d); Shaded areas are SWH biases 

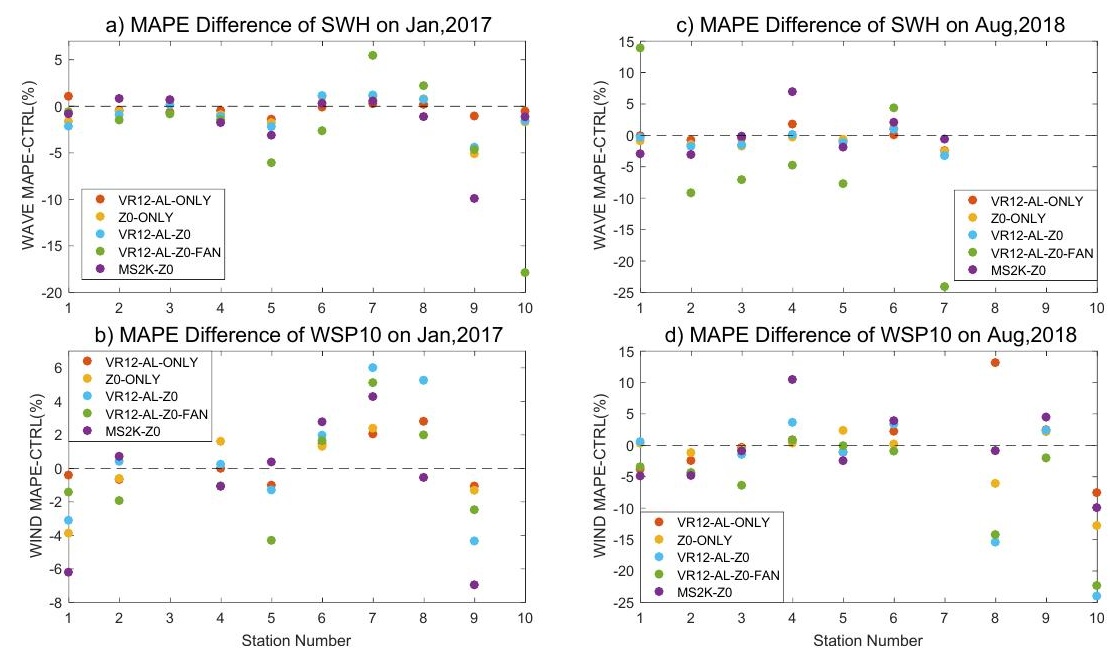

Figure 11. The mean absolute percentage error (MAPE) differences for SWH (a, c) and WSP10 (b, d) between

VR12-AL-ONLY/Z0-ONLY/VR12-AL-Z0/VR12-AL-Z0-FAN/MS2K-Z0 and CTRL (MAPE in VR12-AL-

ONLY/Z0-ONLY/VR12-AL-Z0/VR12-AL-Z0-FAN/MS2K-Z0 minus MAPE in CTRL) on Jan, 2017 (a, b) and Aug, 


\section{Summary and Discussion}

To investigate the role played by ocean surface gravity waves on atmosphere and ocean interface in a generating strong vertical movements for 7-day forecasting. It is beneficial for areas with large errors of SST, such as the ACC. Particularly, the application of VR12-AL parameterization (Van et al. 2012) could significantly reduce the warm bias of SST and shallow bias of MLD in ACC in January, whereas its effects are nil in August. In contrast, the vertical mixing generated by MS2K parameterization is so strong that the SST is too cold and the MLD is too deep 
compared with the observations.

2. With the application of ST4-FAN (Fan et al. 2012) for surface roughness length $\left(z_{0}\right), z_{0}$ becomes larger at high wind conditions, and leads to increased frictional dissipation at oceanatmosphere interface. As a result, the overestimated wind speeds (usually in mid-high latitudes) are reduced. The reduced wind speeds subsequently decrease SWHs, and thus the overestimated SWHs produced by previously overestimated wind are also reduced.

As shown in Fig. 3, the Langmuir mixing induced SST improvements are mainly distributed in mid-high latitudes. SST biases also appeared in tropical oceans. In the work of Chune et al. (2018), the Nucleus for European Modelling of the Ocean (NEMO) model was one-way coupled with the Météo-France wave model (MFWAM) to refine the momentum as well as the energy flux across the air-sea interface. Consequently, the SST cold bias in the tropics is reduced. This offers a next direction to improve the global ocean forecast. Besides, some other processes such as nonbreaking wave-induced upper ocean mixing (Qiao et al., 2004), may also lead to improvements.

There still remain some biases in the coupled system, probably owing to the inaccuracy of coarse resolution, the incompleteness of direct wave-current interaction processes, and the deficiency of a unified assimilation system. In addition, to further improve the model and eliminate the biases, as Breivik et al. (2015) proposed, extra adjusting of the individual model components in the coupled systems is also necessary. All of these require further efforts to investigate efficient methods that can improve the ability of the fully coupled system.

\section{Code and data availability}

The code developed for the coupled system can be found under https://doi.org/10.5281/zenodo.4125726

(Shi et al., 2020), including the coupling, preprocessing, run control and postprocessing scripts. The 
461 initial fields for CFS are generated by the real time operational Climate Data Assimilation System,

462 downloaded from the CFS official website (http://nomads.ncep.noaa.gov/pub/data/nccf/com/cfs/prod).

463 The daily average satellite Optimum Interpolation SST (OISST) data are obtained from NOAA

464 (https://www.ncdc.noaa.gov/oisst), and the National Data Buoy Center (NDBC) buoy data are also

465 obtained from NOAA (https://www.ndbc.noaa.gov). The Argo observational profiles of temperature and

466 salinity are available at China Argo Real-time Data Center (www.argo.org.cn). The ERA5 reanalysis are

467 available at the Copernicus Climate Change Service (C3S) Climate Date Store

Jason-3 satellite data are obtained from AVISO CNES Data Center (https://aviso-data-center.cnes.fr).

\section{Author contribution} and editing. 

http://dx.doi.org/10.1175/2010BAMS2946.1, 2011. 2012. Bidlot, J.-R.: Present status of wave forecasting at ECMWF, Workshop on ocean waves, 2012, 25-27. the NEMO ocean model: Forced and coupled experiments, Journal of Geophysical Research, 120, 29732992, http://dx.doi.org/10.1002/2014JC010565, 2015. 

http://dx.doi.org/10.1175/JCLI-D-11-00621.1, 2012. http://dx.doi.org/10.1175/JCLI-D-13-00583.1, 2014. 

0477(1996)077\%3C0437:TNYRP\%3E2.0.CO;2, 1996. 2008.

Kukulka, T., Plueddemann, A. J., Trowbridge, J. H., and Sullivan, P. P.: Significance of Langmuir circulation in upper ocean mixing : comparison of observations and simulations, Geophysical Research Letters, 36, http://dx.doi.org/10.1029/2009GL037620, 2009.

Li, M., and Garrett, C.: Mixed Layer Deepening Due to Langmuir Circulation, Journal of Physical 0485(1997)027\%3C0121:MLDDTL\%3E2.0.CO;2, 1997. 160, http://dx.doi.org/10.1016/j.ocemod.2015.07.020, 2016.

541 Li, Q., Foxkemper, B., Breivik, O., and Webb, A.: Statistical models of global Langmuir mixing, Ocean Modelling, 113, 95-114, http://dx.doi.org/10.1016/j.ocemod.2017.03.016, 2017. 

http://dx.doi.org/10.5194/gmd-11-3557-2018, 2018. Technology Bulletin, 6, 225-237, http://dx.doi.org/10.1016/S1353-2561(01)00041-X, 2000. Moon, I., Ginis, I., and Hara, T.: Effect of surface waves on Charnock coefficient under tropical cyclones, Geophysical Research Letters, 31, http://dx.doi.org/10.1029/2004GL020988, 2004. Moum J.N., and Smyth W.D.: Upper Ocean Mixing. In Cochran, J. Kirk; Bokuniewicz, J. Henry; Yager, in Modeling Earth Systems, 10, 801-825, http://dx.doi.org/10.1002/2017MS001177, 2018. 

Society, 91, 1015-1057, http://dx.doi.org/10.1175/2010BAMS3001.1, 2010. http://dx.doi.org/10.1175/JCLI-D-12-00823.1, 2014. 
586 Sun, J., Wei, Z., Xu, T., Sun, M., Liu, K., Yang, Y., Chen, L., Zhao, H., Yin, X., and Feng, W.:

587 Development of a fine-resolution atmosphere-wave-ocean coupled forecasting model for the South

China Sea and its adjacent seas, Acta Oceanologica Sinica, 38, 154-166, http://dx.doi.org/10.1175/1520-0485(2001)031\%3C0572:TDOSSR\%3E2.0.CO;2, 2001. 0485(1996)026\%3C0792:EOKEDU\%3E2.0.CO;2, 1996. 0485(1996)026\%3C2497:STIATG\%3E2.0.CO;2, 1996. http://dx.doi.org/10.1016/j.ocemod.2010.07.010, 2010. 
https://doi.org/10.5194/gmd-2020-327

Preprint. Discussion started: 29 October 2020

(c) Author(s) 2020. CC BY 4.0 License.

608 Wu, Z., Jiang, C., Chen, J., Long, Y., Deng, B., and Liu, X.: Three-Dimensional Temperature Field

609 Change in the South China Sea during Typhoon Kai-Tak (1213) Based on a Fully Coupled Atmosphere-

610 Wave-Ocean Model, Water, 11, 140, http://dx.doi.org/10.3390/w11010140, 2019.

611 Zieger, S., Babanin, A. V., Rogers, W. E., and Young, I. R.: Observation-based source terms in the third-

612 generation wave model WAVEWATCH, Ocean Modelling, 96, 2-25,

613 http://dx.doi.org/10.1016/j.ocemod.2015.07.014, 2015.

614 